\title{
Some New Aspects of the Coupon-Collector's Problem
}

\author{
Amy N. Myers and Herbert S. Wilf \\ University of Pennsylvania \\ Philadelphia, PA 19104-6395
}

October 24,2018

\begin{abstract}
We extend the classical coupon collector's problem to one in which two collectors are simultaneously and independently seeking collections of $d$ coupons. We find, in finite terms, the probability that the two collectors finish at the same trial, and we find, using the methods of Gessel-Viennot, the probability that the game has the following "ballotlike" character: the two collectors are tied with each other for some initial number of steps, and after that the player who first gains the lead remains ahead throughout the game. As a by-product we obtain the evaluation in finite terms of certain infinite series whose coefficients are powers and products of Stirling numbers of the second kind.

We study the variant of the original coupon collector's problem in which a single collector wants to obtain at least $h$ copies of each coupon. Here we give a simpler derivation of results of Newman and Shepp, and extend those results. Finally we obtain the distribution of the number of coupons that have been obtained exactly once ("singletons") at the conclusion of a successful coupon collecting sequence.
\end{abstract}




\section{Contents}

1 Introduction and resuits $\quad 2$

2 The two-person collecting competition 3

Z.1 Simuitaneous completion . . . . . . . . . . . . . . . . . . . . . . . . . . . . . . . 3

Z.2 Neck-and-neck, then alwavs ahead . . . . . . . . . . . . . . . . . . . . . . . . . . . . 5

Z.3 The case $k<n-z$. . . . . . . . . . . . . . . . . . . . . . . . . . . . . . . . . . . . . 5

2.4 Paths from Ato $B$. . . . . . . . . . . . . . . . . . . . . . . . . . . . . . . . . . . . . 7

Z.5 Evaluating the determinant . . . . . . . . . . . . . . . . . . . . . . . . . . . . . . . . . . . . . . . . . . . .

2.0 The initial common segment . . . . . . . . . . . . . . . . . . . . . . . . . . . . . . . . 9

Z.T The case $k=n-1$. . . . . . . . . . . . . . . . . . . . . . . . . . . 9

2.8 Putting it togethen . . . . . . . . . . . . . . . . . . . . . . . . . . . . . . . . . . . . . 10

Z.9 One collector never behind . . . . . . . . . . . . . . . . . . . . . . . . . . . . . . . . 11

3 The "double dixie-cup probiem," of Newman and Shepp, revisited 14

3.1 Two examples . . . . . . . . . . . . . . . . . . . . . . . . . 16

3.2 Asvmptotics . . . . . . . . . . . . . . . . . . . . . . . . . . . . . 17

4 The number of singletons 18

References 20

\section{Introduction and results}

The classical coupon collector's problem is the following. Suppose that a breakfast cereal manufacturer offers a souvenir ("coupon") hidden in each package of cereal, and there are $d$ different kinds of souvenirs altogether. The collector wants to have a complete collection of all $d$ souvenirs. What is the probability $p(n, d)$ that exactly $n$ boxes of cereal will have to be purchased in order to obtain, for the first time, a complete collection of at least one of each of the $d$ kinds of souvenir coupons?

The answer to that question is well known (e.g., [6], p. 132) to be

$$
p(n, d)=\frac{d !}{d^{n}}\left\{\begin{array}{l}
n-1 \\
d-1
\end{array}\right\},
$$

where the $\left\{\begin{array}{l}n \\ k\end{array}\right\}$ 's are the Stirling numbers of the second kind.

We study, in this paper, a number of other aspects of this problem, as well as a generalization of it to a two-player game.

First, suppose we have two coupon collectors, drawing coupons simultaneously, and each seeking to obtain a complete collection of $d$ coupons. We ask for the probability that the two games are completed at the same time. The answer is given by (17) below. That answer is expressed in finite terms, owing to the closed form evaluation of the ordinary power series 
generating function for the squares of the Stirling numbers of the second kind, contained in (6).

Next we consider the following two-person game. Again two coupon collectors are simultaneously drawing coupons at random. This time we are interested in a ballot-like problem: what is the probability that the player who first completed a collection (the winner) was never behind (i.e., never had fewer distinct coupons) at any intermediate stage of the play? Here we give a complete answer to a slightly easier question, namely the following: what is the probability that after an initial segment of play in which the players are tied, one of them takes the lead and keeps the lead until the end. The answer is in eq. (27) below, and is obtained by the Gessel-Viennot theory of nonintersecting lattice paths.

In each of these cases the answer can first be written as an infinite series whose coefficients involve various products of Stirling numbers. What is interesting, though, is that in all such cases we are able to express the answers in finite terms. Indeed, one of our main results here is the observation that infinite series whose coefficients involve various powers and products of Stirling numbers of the second kind can readily be evaluated in finite terms.

In section 3 we return to the original collecting problem of obtaining at least one copy of each coupon, but now we study the variant of the problem in which a single collector wants to obtain at least $h \geq 1$ copies of each coupon. We obtain the generating function (33) for the probability that exactly $n$ trials are needed, the exact value of the average number of trials (38), and the asymptotic behavior (43) of these quantities as $n \rightarrow \infty$.

Finally, in section 4 we study the number of coupons that have been collected only once, at the end of a collection sequence. We find the distribution function (47) for this number, and show that the average number of these singletons is just the harmonic number $H_{d}=1+1 / 2+\ldots+1 / d$.

\section{The two-person collecting competition}

\subsection{Simultaneous completion}

We find now the probability of simultaneous completion of two independent coupon collecting sequences. Evidently this is,

$$
\sum_{n \geq 0} p(n, d)^{2}=\sum_{n \geq 0} \frac{d !^{2}}{d^{2 n}}\left\{\begin{array}{l}
n-1 \\
d-1
\end{array}\right\}^{2},
$$

which expresses the answer as an infinite sum. We can rewrite this as a finite sum by finding a finite expression for the generating function for the squares of the Stirling numbers of the 
second kind,

$$
F_{k}(x) \stackrel{\text { def }}{=} \sum_{n \geq k}\left\{\begin{array}{l}
n \\
k
\end{array}\right\}^{2} x^{n},
$$

analogously to the well known generating function for these numbers themselves,

$$
\sum_{n \geq k}\left\{\begin{array}{l}
n \\
k
\end{array}\right\} x^{n}=\frac{x^{k}}{(1-x)(1-2 x) \ldots(1-k x)} .
$$

The easiest way to do this is via the standard explicit formula for these Stirling numbers, viz.

$$
\begin{aligned}
\left\{\begin{array}{l}
n \\
k
\end{array}\right\} & =\frac{1}{k !} \sum_{r=1}^{k}(-1)^{k-r}\left(\begin{array}{l}
k \\
r
\end{array}\right) r^{n} \quad(1 \leq k \leq n) \\
& \stackrel{\text { def }}{=} \sum_{r=1}^{k} A_{k, r} r^{n-k}
\end{aligned}
$$

where we have written

$$
A_{k, r}=\frac{(-1)^{k-r} r^{k}}{k !}\left(\begin{array}{l}
k \\
r
\end{array}\right)
$$

It follows that

$$
\begin{aligned}
F_{k}(x) \stackrel{\text { def }}{=} \sum_{n \geq k}\left\{\begin{array}{l}
n \\
k
\end{array}\right\}^{2} x^{n} & =\sum_{n \geq k} x^{n} \sum_{r, s=1}^{k} A_{k, r} A_{k, s} r^{n-k} s^{n-k} \\
& =x^{k} \sum_{r, s=1}^{k} A_{k, r} A_{k, s} \sum_{n \geq k}(r s x)^{n-k} \\
& =x^{k} \sum_{r, s=1}^{k} \frac{A_{k, r} A_{k, s}}{1-r s x}, \quad\left(|x|<\frac{1}{k^{2}}\right) .
\end{aligned}
$$

Thus for the simultaneous completion probability we obtain, from (2),

$$
\sum_{n \geq 0} p(n, d)^{2}=\frac{d !^{2}}{d^{2 d}} \sum_{r, s=1}^{d-1} \frac{A_{d-1, r} A_{d-1, s}}{1-\frac{r s}{d^{2}}},
$$

by (6), where the $A$ 's given by (5). This sequence of probabilities, for $d=1,2, \ldots$, begins as

$$
1, \frac{1}{3}, \frac{11}{70}, \frac{9}{91}, \frac{688877}{9561123}, \frac{358555}{6330324}, \frac{2730269557627901}{58560931675094420}, \frac{146271649897951}{3695016639410525}, \ldots,
$$
i.e., as

$$
\text { 1,0.33333..,0.15714..,0.098901..,0.072049..,0.056640..,0.046622..,0.039586..,... }
$$




\subsection{Neck-and-neck, then always ahead}

We encode a sequence of $n$ draws as a path $\omega$ with $n$ vertices in the lattice $\mathcal{L}$ consisting of vertices $(i, j)$, and edges $\{(i, j),(i+1, j)\},\{(i, j),(i+1, j+1)\}$, for all $i, j \geq 0$. The first coordinate of a vertex in the path gives the number of draws, or steps, and the second coordinate gives the number of distinct coupons the collector has at that step. Thus $\omega$ starts at $(0,0)$ indicating the collector has 0 coupons at draw 0 , proceeds to $(1,1)$ (the collector has 1 coupon after 1 draw), and ends at $(n, d), n \geq d$ (the collector has a complete collection at step $n)$. We write $\omega=(0,0) \bar{\omega}(n, d)$, where $\bar{\omega}$ is a path from $(1,1)$ to $(n-1, d-1)$, to indicate that $\omega$ starts at the vertex $(0,0)$, continues with the first vertex $(1,1)$ in $\bar{\omega}$, then follows $\bar{\omega}$ through to $(n-1, d-1)$, and finally ends with the vertex $(n, d)$.

We assign a weight of $i / d$ to each horizontal edge $\{(i, j),(i+1, j)\}$ in the lattice $\mathcal{L}$. This is the probability that at the $(j+1)^{\text {st }}$ step, the collector draws one of the $i$ distinct coupons already collected at step $j$. We assign a weight of $1-i / d$ to each northeast edge $\{(i, j),(i+1, j+1)\}$. The probability that the collector draws the particular sequence of coupons encoded by the path $\omega$ is given by the product of the weights on the edges of $\omega$. We let $P(\omega)$ denote this probability.

Suppose one collector, the winner, collects all $d$ distinct coupons for the first time at step $n$. (At step $n-1$ the winner had $d-1$ distinct coupons.) Let $\omega_{1}$ be the lattice path which encodes the winner's sequence of draws. Let $\omega_{2}$ encode the other collector's draws. We compute the probability $p(d)$ that $\omega_{1}$ and $\omega_{2}$ are identical until some point at which the winner takes the lead and the other collector never catches up.

To do this, we begin by supposing $\omega_{1}$ is identical to $\omega_{2}$ until step $k$, at which point both collectors have $d_{1}$ distinct coupons. The argument splits into two cases, namely $k \leq n-2$ and $k=n-1$. In both cases, at step $k+1$ the winner collects one additional distinct coupon while the other collector does not. After step $k$, the two paths never intersect again. The winner collects all $d$ distinct coupons for the first time at step $n$. Suppose the other collector has $d_{2}$ distinct coupons at this point. The probability we seek is

$$
p(d)=\sum_{n=d}^{\infty} \sum_{k=1}^{n-1} \sum_{d_{1}=1}^{d-1} \sum_{d_{2}=d_{1}}^{d-1} \sum_{\left(\omega_{1}, \omega_{2}\right)} P\left(\omega_{1}\right) P\left(\omega_{2}\right)
$$

where the innermost sum ranges over all pairs $\left(\omega_{1}, \omega_{2}\right)$ described above.

\subsection{The case $k \leq n-2$}

Write $\omega_{1}=\alpha \overline{\omega_{1}}(n, d)$, where $\alpha$ denotes a lattice path from $(0,0)$ to $\left(k, d_{1}\right)$, and $\overline{\omega_{1}}$ denotes a path from $\left(k+1, d_{1}+1\right)$ to $(n-1, d-1)$. Similarly, set $\omega_{2}=\alpha \overline{\omega_{2}}$, where $\alpha$ is as 
above, and $\overline{\omega_{2}}$ is a path from $\left(k+1, d_{1}\right)$ to $\left(n, d_{2}\right)$. Note that $\overline{\omega_{1}}$ and $\overline{\omega_{2}}$ are nonintersecting paths in the lattice $\mathcal{L}$. In terms of these we have $P\left(\omega_{1}\right)=P(\alpha)\left(1-d_{1} / d\right) P\left(\overline{\omega_{1}}\right)(1 / d)$ and $P\left(\omega_{2}\right)=P(\alpha)\left(d_{1} / d\right) P\left(\overline{\omega_{2}}\right)$. Hence from (8) we find for the combined probability of all pairs if $k \leq n-2$,

$$
\begin{aligned}
p(d)_{k \leq n-2} & =\sum_{n=d}^{\infty} \sum_{k=1}^{n-2} \sum_{d_{1}=1}^{d-1} \sum_{d_{2}=d_{1}}^{d-1} \sum_{\left(\omega_{1}, \omega_{2}\right)}\left[P(\alpha)\left(1-\frac{d_{1}}{d}\right) P\left(\overline{\omega_{1}}\right)\left(\frac{1}{d}\right)\right]\left[P(\alpha)\left(\frac{d_{1}}{d}\right) P\left(\overline{\omega_{2}}\right)\right] \\
& =\sum_{n=d}^{\infty} \sum_{k=1}^{n-2} \sum_{d_{1}=1}^{d-1} \sum_{d_{2}=d_{1}}^{d-1}\left(1-\frac{d_{1}}{d}\right)\left(\frac{1}{d}\right)\left(\frac{d_{1}}{d}\right) \sum_{\alpha} P(\alpha)^{2} \sum_{\left(\overline{\omega_{1}}, \overline{\omega_{2}}\right)} P\left(\overline{\omega_{1}}\right) P\left(\overline{\omega_{2}}\right)
\end{aligned}
$$

At this point we have translated a question about coupon collecting into a problem involving nonintersecting paths in a lattice. We have set the stage for application of the Gessel-Viennot theorem [4. This result concerns pairs of nonintersecting lattice paths with no constraints on vertices or edges in the paths. For this reason we have written $\omega_{1}$ and $\omega_{2}$ in terms of $\overline{\omega_{1}}$ and $\overline{\omega_{2}}$.

The theorem refers to an arbitrary set $\mathcal{L}$, which we will take to be the lattice defined earlier, and a weight (or valuation) $v$, which we take to be $P$. The theorem equates a sum of weights of paths with the determinant of a matrix $\left(a_{i j}\right)_{1 \leq i, j \leq l}$. The entries of this matrix are defined by $a_{i j}=\sum_{\omega} v(\omega)$, where $\omega$ ranges over all paths from $A_{i}$ to $B_{j}$.

The theorem requires that two given sequences, $\left(A_{1}, A_{2}, \ldots, A_{l}\right)$ and $\left(B_{1}, B_{2}, \ldots, B_{l}\right)$, of vertices in $\mathcal{L}$, the sets $\Omega_{i j}, 1 \leq i, j \leq l$, of all paths in $\mathcal{L}$ between $A_{i}$ and $B_{j}$, and the weight $v$ satisfy both the finiteness and crossing conditions. The finiteness condition requires the set of paths in $\Omega_{i j}$ with nonzero weight be finite. The crossing condition requires that paths in $\Omega_{i j^{\prime}}$ and $\Omega_{i^{\prime} j}, i<i^{\prime}$ and $j<j^{\prime}$, with nonzero weight share a common vertex. Both conditions hold for the paths we consider.

Theorem 1 (Gessel-Viennot) Suppose $\mathcal{L}, v,\left(A_{1}, A_{2}, \ldots, A_{l}\right)$, and $\left(B_{1}, B_{2}, \ldots, B_{l}\right)$ satisfy both the finiteness and crossing conditions. Then the determinant of the matrix $\left(a_{i j}\right)_{1 \leq i, j \leq l}$ is the sum of the weights of all configurations of paths $\left(\omega_{1}, \omega_{2}, \ldots, \omega_{l}\right)$ satisfying the following two conditions:

(i) The paths $\omega_{k}$ are pairwise nonintersecting, and

(ii) $\omega_{k}$ is a path from $A_{k}$ to $B_{k}$.

In other words,

$$
\operatorname{det}\left(\left\{a_{i j}\right\}_{i, j=1}^{l}\right)=\sum_{\left(\omega_{1}, \omega_{2}, \ldots, \omega_{l}\right)} v\left(\omega_{1}\right) v\left(\omega_{2}\right) \ldots v\left(\omega_{l}\right) .
$$


Application of this theorem to our problem requires the computation of only a $2 \times 2$ determinant! Let $A_{1}=\left(k+1, d_{1}+1\right), A_{2}=\left(k+1, d_{1}\right), B_{1}=(n-1, d-1)$, and $B_{2}=\left(n, d_{2}\right)$. Then

$$
\sum_{\left(\overline{\omega_{1}}, \overline{\omega_{2}}\right)} P\left(\overline{\omega_{1}}\right) P\left(\overline{\omega_{2}}\right)=\operatorname{det}\left[\begin{array}{ll}
a_{11} & a_{12} \\
a_{21} & a_{22}
\end{array}\right]
$$

where $a_{i j}$ is the sum $\sum_{\omega} P(\omega)$ over all paths $\omega$ from $A_{i}$ to $B_{j}$.

\subsection{Paths from $A$ to $B$}

In this section, we compute the probability $P(\omega)$ of an arbitrary path $\omega$ from a vertex $A=\left(a_{1}, b_{1}\right)$ to a vertex $B=\left(a_{2}, b_{2}\right)$, as well as the sum over all such paths. Such a path contains $b_{2}-b_{1}$ northeast edges $\{(i, j),(i+1, j+1)\}$ and $\left(a_{2}-a_{1}\right)-\left(b_{2}-b_{1}\right)$ horizontal edges. The weights assigned to northeast edges in order from left to right are $1-\frac{b_{1}}{d}, 1-\frac{b_{1}+1}{d}, \ldots, 1-$ $\frac{b_{2}-1}{d}$. The weight assigned to a horizontal edge depends its coordinates. Consider the edge $\{(i, j),(i+1, j)\}$. This edge indicates the collector has $j$ distinct coupons at step $i$ and draws one of the same $j$ coupons at step $i+1$. The probability of this (weight of the edge) is $\frac{j}{d}$. Thus the probability of a path $\omega$ from $A$ to $B$ is

$$
\begin{aligned}
P(\omega) & =\left(\frac{b_{1}}{d}\right)^{e_{1}}\left(1-\frac{b_{1}}{d}\right)\left(\frac{b_{1}+1}{d}\right)^{e_{2}}\left(1-\frac{b_{1}+1}{d}\right) \ldots\left(1-\frac{b_{2}-1}{d}\right)\left(\frac{b_{2}}{d}\right)^{e_{b_{2}-b_{1}+1}} \\
& =\frac{1}{d^{a_{2}-a_{1}}} \frac{\left(d-b_{1}\right) !}{\left(d-b_{2}\right) !}\left(b_{1}\right)^{e_{1}}\left(b_{1}+1\right)^{e_{2}} \ldots\left(b_{2}\right)^{e_{b_{2}-b_{1}+1}}
\end{aligned}
$$

where $e=\left(e_{1}, e_{2}, \ldots, e_{b_{2}-b_{1}+1}\right)$ is an ordered partition, a composition, of $\left(a_{2}-a_{1}\right)-\left(b_{2}-b_{1}\right)$ into $b_{2}-b_{1}+1$ nonnegative integer parts. With this we compute the sum of the probabilities of all paths from $A$ to $B$.

$$
\begin{aligned}
\sum_{\omega=A \cdots B} P(\omega) & =\sum_{e}\left(\frac{b_{1}}{d}\right)^{e_{1}}\left(1-\frac{b_{1}}{d}\right)\left(\frac{b_{1}+1}{d}\right)^{e_{2}}\left(1-\frac{b_{1}+1}{d}\right) \ldots\left(1-\frac{b_{2}-1}{d}\right)\left(\frac{b_{2}}{d}\right)^{e_{b_{2}-b_{1}+1}} \\
& =\frac{1}{d^{a_{2}-a_{1}}} \frac{\left(d-b_{1}\right) !}{\left(d-b_{2}\right) !} \sum_{e}\left(b_{1}\right)^{e_{1}}\left(b_{1}+1\right)^{e_{2}} \ldots\left(b_{2}\right)^{e_{b_{2}-b_{1}+1}}
\end{aligned}
$$

where the sum is over all compositions $e=\left(e_{1}, e_{2}, \ldots, d_{b_{2}-b_{1}+1}\right)$ of $\left(a_{2}-a_{1}\right)-\left(b_{2}-b_{1}\right)$ into $b_{2}-b_{1}+1$ nonnegative integer parts. This is the coefficient of $x^{\left(a_{2}-a_{1}\right)-\left(b_{2}-b_{1}\right)}$ in the series expansion of

$$
\frac{1}{\left(1-b_{1} x\right)\left(1-\left(b_{1}+1\right) x\right) \ldots\left(1-b_{2} x\right)}
$$


so we can find a simpler formula for it by looking at the partial fraction expansion

$$
\frac{1}{\prod_{m=a}^{b}(1-m x)}=\sum_{m=a}^{b} \frac{B_{m}}{1-m x},
$$

where

$$
B_{m}=\frac{(-1)^{b-m} m^{b-a}}{(b-a) !}\left(\begin{array}{c}
b-a \\
m-a
\end{array}\right) .
$$

From this and (12) we obtain

$$
\begin{aligned}
\sum_{\omega=A \cdots B} P(\omega) & =\frac{1}{d^{a_{2}-a_{1}}} \frac{\left(d-b_{1}\right) !}{\left(d-b_{2}\right) !}\left[x^{\left(a_{2}-a_{1}\right)-\left(b_{2}-b_{1}\right)}\right]\left\{\sum_{m=b_{1}}^{b_{2}} \frac{B_{m}}{1-m x}\right\} \\
& =\frac{1}{d^{a_{2}-a_{1}}} \frac{\left(d-b_{1}\right) !}{\left(d-b_{2}\right) !} \sum_{m=b_{1}}^{b_{2}} B_{m} m^{\left(a_{2}-a_{1}\right)-\left(b_{2}-b_{1}\right)} \\
& =\frac{1}{d^{a_{2}-a_{1}}} \frac{\left(d-b_{1}\right) !}{\left(d-b_{2}\right) !} \sum_{m=b_{1}}^{b_{2}} \frac{(-1)^{b_{2}-m} m^{a_{2}-a_{1}}}{\left(b_{2}-b_{1}\right) !}\left(\begin{array}{c}
b_{2}-b_{1} \\
m-b_{1}
\end{array}\right)
\end{aligned}
$$

\subsection{Evaluating the determinant}

We use the results of the previous section to evaluate the determinant in (10). To compute $a_{11}$, we substitute $A=A_{1}=\left(k+1, d_{1}+1\right)$ and $B=B_{1}=(n-1, d-1)$ in (14). This yields

$$
a_{11}=\frac{1}{d^{n-k-2}}\left(d-d_{1}-1\right) ! \sum_{m=d_{1}+1}^{d-1} \frac{(-1)^{d-m-1} m^{n-k-2}}{\left(d-d_{1}-2\right) !}\left(\begin{array}{c}
d-d_{1}-2 \\
m-d_{1}-1
\end{array}\right)
$$

In a similar manner we obtain

$$
\begin{aligned}
& a_{12}=\frac{1}{d^{n-k-1}} \frac{\left(d-d_{1}-1\right) !}{\left(d-d_{2}\right) !} \sum_{m=d_{1}+1}^{d_{2}} \frac{(-1)^{d_{2}-m} m^{n-k-1}}{\left(d_{2}-d_{1}-1\right) !}\left(\begin{array}{c}
d_{2}-d_{1}-1 \\
m-d_{1}-1
\end{array}\right) \\
& a_{21}=\frac{1}{d^{n-k-2}}\left(d-d_{1}\right) ! \sum_{m=d_{1}}^{d-1} \frac{(-1)^{d-m-1} m^{n-k-2}}{\left(d-d_{1}-1\right) !}\left(\begin{array}{c}
d-d_{1}-1 \\
m-d_{1}
\end{array}\right) \\
& a_{22}=\frac{1}{d^{n-k-1}} \frac{\left(d-d_{1}\right) !}{\left(d-d_{2}\right) !} \sum_{m=d_{1}}^{d_{2}} \frac{(-1)^{d_{2}-m} m^{n-k-1}}{\left(d_{2}-d_{1}\right) !}\left(\begin{array}{c}
d_{2}-d_{1} \\
m-d_{1}
\end{array}\right)
\end{aligned}
$$

Using (15)-(18) we compute the determinant of our $2 \times 2$ matrix.

$\operatorname{det}\left[\begin{array}{ll}a_{11} & a_{12} \\ a_{21} & a_{22}\end{array}\right]=\frac{\left(d-d_{1}\right) !\left(d-d_{1}-1\right) !}{d^{2 n-2 k-3}\left(d-d_{2}\right) !} \times$ 


$$
\begin{aligned}
& \stackrel{\sum_{l=d_{1}}}{\stackrel{d_{2}}{=}} \quad \begin{array}{l}
\operatorname{det}\left(d, d_{1}, d_{2}, k, n\right) \\
\left(d-d_{1}-1\right) !\left(d_{2}-d_{1}\right) !
\end{array}\left(\begin{array}{c}
d-d_{1}-1 \\
l-d_{1}
\end{array}\right)\left(\begin{array}{c}
d_{2}-d_{1} \\
m-d_{1}
\end{array}\right) \\
&
\end{aligned}
$$

Substituting (201) in (10), we obtain

$$
\sum_{\left(\overline{\omega_{1}}, \overline{\omega_{2}}\right)} P\left(\overline{\omega_{1}}\right) P\left(\overline{\omega_{2}}\right)=\operatorname{det}\left(d, d_{1}, d_{2}, k, n\right)
$$

\subsection{The initial common segment}

In the previous section we evaluated the determinant in (10). In this section we compute the sum $\sum_{\alpha} P(\alpha)^{2}$ in (9). Recall $\alpha$ is a path from $(0,0)$ to $\left(k, d_{1}\right)$.

Equation (11) gives the probability of an arbitrary path from $A$ to $B$. Substituting $A=(0,0)$ and $B=\left(k, d_{1}\right)$ gives the probability

$$
P(\alpha)=\frac{d !}{d^{k}\left(d-d_{1}\right) !} 1^{e_{1}} 2^{e_{2}} \cdots d_{1} e_{d_{1}}
$$

of an arbitrary path $\alpha$ from $(0,0)$ to $\left(k, d_{1}\right)$. It follows that

$$
\begin{aligned}
\sum_{\alpha=(0,0) \cdots\left(k, d_{1}\right)} P(\alpha)^{2} & =\frac{d !^{2}}{d^{2 k}\left(d-d_{1}\right) !^{2}} \sum_{e_{1}+\ldots+e_{d_{1}}=k-d_{1}} 1^{2 e_{1}} 2^{2 e_{2}} \ldots d_{1}^{2 e_{d_{1}}} \\
& =\frac{d !^{2}}{d^{2 k}\left(d-d_{1}\right) !^{2}}\left[x^{k-d_{1}}\right]\left\{\frac{1}{\left(1-1^{2} x\right)\left(1-2^{2} x\right) \ldots\left(1-d_{1}^{2} x\right)}\right\} \\
& =\frac{d !^{2}}{d^{2 k}\left(d-d_{1}\right) !^{2}} \sum_{m=1}^{d_{1}} C_{m} m^{2 k-2 d_{1}} \quad(\text { as in (13) }) \\
& =\frac{2 d !^{2}}{\left(d-d_{1}\right) !^{2} d^{2 k}\left(2 d_{1}\right) !} \sum_{m \geq 1}(-1)^{d_{1}-m}\left(\begin{array}{c}
2 d_{1} \\
d_{1}+m
\end{array}\right) m^{2 k} . \\
& \stackrel{\text { def }}{=} \operatorname{init~}\left(d, d_{1}, k\right)
\end{aligned}
$$

\subsection{The case $k=n-1$}

Suppose now that the two walks are identical up to the point $(n-1, d-1)$. Since step $n$ is the finish, the next step for the winning player will be to $(n, d)$, and for the loser, to $(n, d-1)$. These last steps have respective probabilities $1 / d$ and $1-1 / d$. Hence the probability of the complete pair of walks in this case is the probability of two identical walks from $(0,0)$ to $(n-1, d-1)$ (which is given by (22) with $\left.\left(k, d_{1}\right):=(n-1, d-1)\right)$ multiplied by $(d-1) / d^{2}$. 


\subsection{Putting it together}

We now substitute (201) and (23) into (9) to obtain the probability of all pairs of paths that we are considering,

$$
\begin{gathered}
p(d)=\sum_{n=d}^{\infty} \sum_{k=1}^{n-2} \sum_{d_{1}=1}^{d-1} \sum_{d_{2}=d_{1}}^{d-1}\left(1-\frac{d_{1}}{d}\right)\left(\frac{d_{1}}{d}\right)\left(\frac{1}{d}\right) \operatorname{init}\left(d, d_{1}, k\right) \operatorname{det}\left(d, d_{1}, d_{2}, k, n\right) \\
+\frac{d-1}{d^{2}} \sum_{n=d}^{\infty} \operatorname{init}(d, d-1, n-1), \\
\stackrel{\text { def }}{=} \Sigma_{1}+\Sigma_{2}
\end{gathered}
$$

It turns out that the sums over the indices $d_{2}, n, k$ can all be carried out in explicit closed form. Hence we can obtain an expression which is in finite terms for the total probability.

First, the sum on $d_{2}$ in $\Sigma_{1}$ above can be done in closed form since

$$
\sum_{d_{2}=d_{1}}^{d-1}(-1)^{d_{2}}\left(\begin{array}{l}
d-d_{1} \\
d-d_{2}
\end{array}\right)\left(\begin{array}{c}
d_{2}-d_{1} \\
t-d_{1}
\end{array}\right)=(-1)^{d+1}\left(\begin{array}{c}
d-d_{1} \\
d-t
\end{array}\right)
$$

Next, the remaining sum over the indices $n$ and $k$, in the first summation, $\Sigma_{1}$ is

$$
\psi(d, r, s, t) \stackrel{\text { def }}{=} \sum_{n=d}^{\infty} \sum_{k=1}^{n-2} \frac{r^{2 k} t^{n-k-1} s^{n-k-2}}{d^{2 n}}= \begin{cases}\frac{r^{2 d}\left(d^{3}-2 d^{2}-r^{2} d+3 r^{2}\right)}{d^{2 d-2}\left(d^{2}-r^{2}\right)^{2} s^{2} t}, & \text { if } r^{2}=s t ; \\ \frac{r^{4}(s t)^{d-1}\left(r^{2}-d^{2}\right)+s t r^{2 d}\left(d^{2}-s t\right)}{d^{2 d-2}\left(d^{2}-s t\right)\left(d^{2}-r^{2}\right)\left(r^{2}-s t\right) r^{2} s}, & \text { otherwise. }\end{cases}
$$

The sum over $n$ in $\Sigma_{2}$ is trivial, and so there remain no infinite sums in our final expression for the probability $p(d)$, which is

$$
\begin{gathered}
\sum_{d_{1}=1}^{d-1} \frac{2 d !^{2} d_{1}\left(d-d_{1}\right)}{\left(d-d_{1}\right) !^{2}\left(2 d_{1}\right) !} \sum_{r, s, t \geq 1}(-1)^{d_{1}-r-s-t}(s-t)\left(\begin{array}{c}
2 d_{1} \\
d_{1}+r
\end{array}\right)\left(\begin{array}{c}
d-d_{1}-1 \\
s-d_{1}
\end{array}\right)\left(\begin{array}{c}
d-d_{1} \\
d-t
\end{array}\right) \psi(d, r, s, t) \\
+\frac{4(d-1) d !^{2}}{d^{2 d-2}(2 d-2) !} \sum_{r=1}^{d-1}(-1)^{d-1-r}\left(\begin{array}{c}
2 d-2 \\
d-1+r
\end{array}\right) \frac{r^{2 d-2}}{d^{2}-r^{2}}+\delta_{d, 1}
\end{gathered}
$$

where $\psi$ is given by (26).

This is the probability that the game is of the type we described, namely where the players are tied for some initial segment of trials and then the player who pulls ahead remains ahead

always, expressed as a finite sum (albeit a complicated one!). More precisely, the values of $p(d)$ can be calculated, as rational numbers, with $O\left(d^{4}\right)$ evaluations of the above summand. The exact values of $p(d)$, for $d=1,2,3,4,5, \ldots$ are

$$
\left\{1, \frac{2}{3}, \frac{43}{70}, \frac{986}{2275}, \frac{5672893}{1912246}, \ldots\right\}
$$


As decimals, the values of $\{p(d)\}_{d=1}^{10}$ are

$$
\{1.0,0.66667,0.61429,0.43341,0.29667,0.21177,0.16016,0.12748,0.10551,0.08988\} \text {. }
$$

\subsection{One collector never behind}

In contrast to the problem of staying ahead as soon as the tie is broken, which we have solved in the preceding sections, the problem in which the ultimate winner has never been behind is unsolved.

Suppose the winner collects all $d$ distinct coupons for the first time at step $n$, at which point the other collector has $d^{\prime}<d$ distinct coupons. We discuss the probability $b(d)$ the winner has never been behind. We use $b$ for "ballot" since this version of the problem has a distinct ballot-problem flavor (see []).

Let $w_{1}$ be the lattice path which encodes the winner's sequence of draws. Let $\omega_{2}$ encode the other collector's sequence of draws. Then $b(d)$ is the probability that $\omega_{2}$ does not cross $\omega_{1}$. To say $\omega_{2}$ does not cross $\omega_{1}$ means for each horizontal coordinate $i$ shared by vertices $\left(i, j_{1}\right)$ in $\omega_{1}$ and $\left(i, j_{2}\right)$ in $\omega_{2}$, we have $j_{2} \leq j_{1}$. In the case $j_{2}=j_{1}$, we say $\omega_{1}$ and $\omega_{2}$ intersect at $\left(i, j_{1}\right)=\left(i, j_{2}\right)$. Thus we seek all pairs $\left(\omega_{1}, \omega_{2}\right)$ such that $\omega_{1}$ is a path from $(0,0)$ to $(n, d)$ including the vertex $(n-1, d-1), \omega_{2}$ is a path from $(0,0)$ to $\left(n, d^{\prime}\right)$ for $1 \leq d^{\prime} \leq d$, and $\omega_{2}$ does not cross $\omega_{1}$. Such a pair $\left(\omega_{1}, \omega_{2}\right)$ is illustrated by Figure 1. Note that $\omega_{1}$ and $\omega_{2}$ may intersect several times. The probability we seek is

$$
b(d)=\sum_{n=d}^{\infty} \sum_{d^{\prime}=1}^{d-1} \sum_{\left(\omega_{1}, \omega_{2}\right)} P\left(\omega_{1}\right) P\left(\omega_{2}\right)
$$

where the innermost sum ranges over all pairs described above.

Look again at Figure 1. A pair $\left(\omega_{1}, \omega_{2}\right)$ appears to form a chain of flying kites anchored to the ground at $(0,0)$. The highest kite has two ribbons attached to its tip. Their loose ends are at $(n, d)$ and $\left(n, d^{\prime}\right)$.

Each kite consists of a frame together with a tail. See Figure 2, A frame from $\left(i_{1}, j_{1}\right)$ to $\left(i_{2}, j_{2}\right)$ consists of a pair of paths from $\left(i_{1}, j_{1}\right)$, the lower tip of the frame, to $\left(i_{2}, j_{2}\right)$, the

upper tip, which intersect only at the endpoints. A tail from $\left(i_{1}, j_{1}\right)$ to $\left(i_{2}, j_{2}\right)$ consists of 


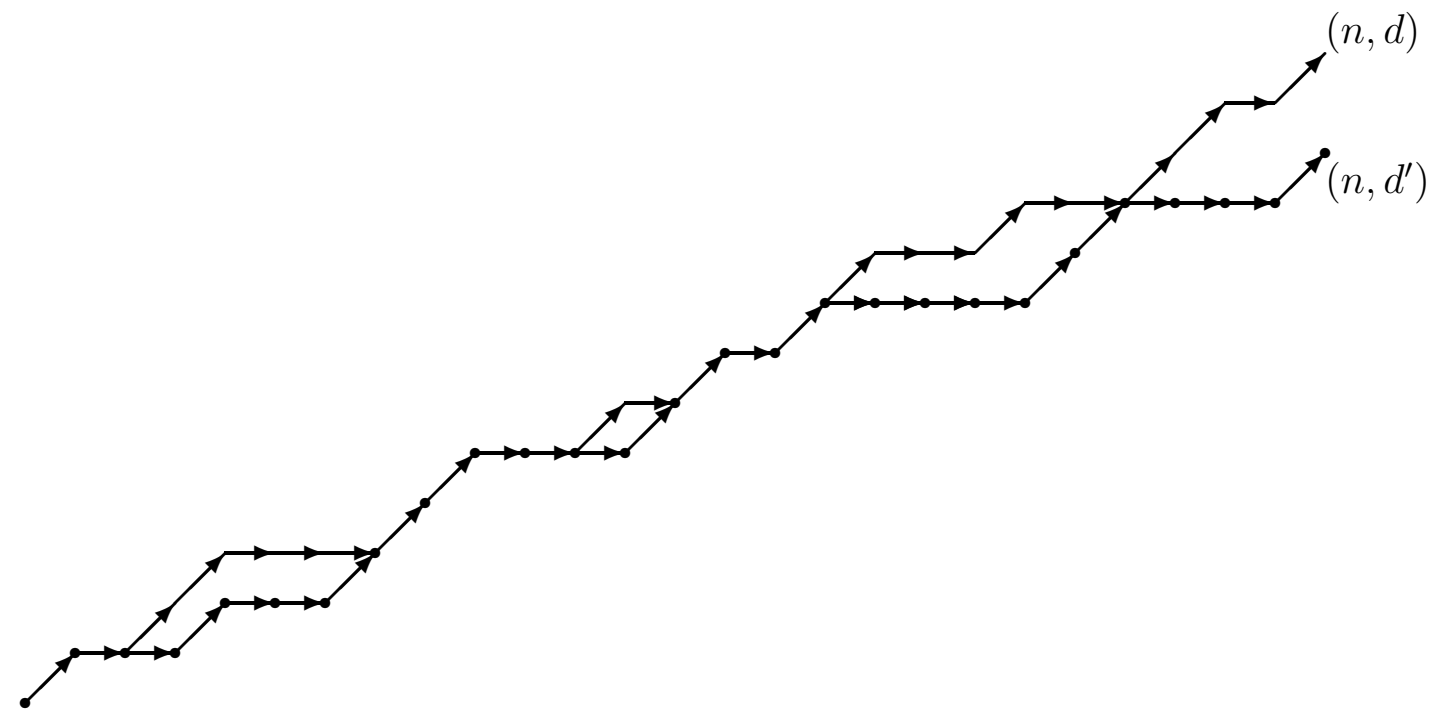

Figure 1: The winner is never behind

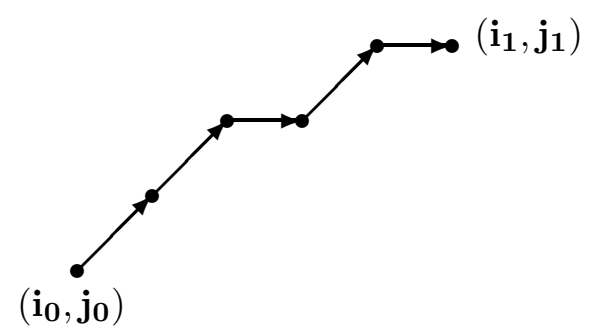

A tail, plus ..
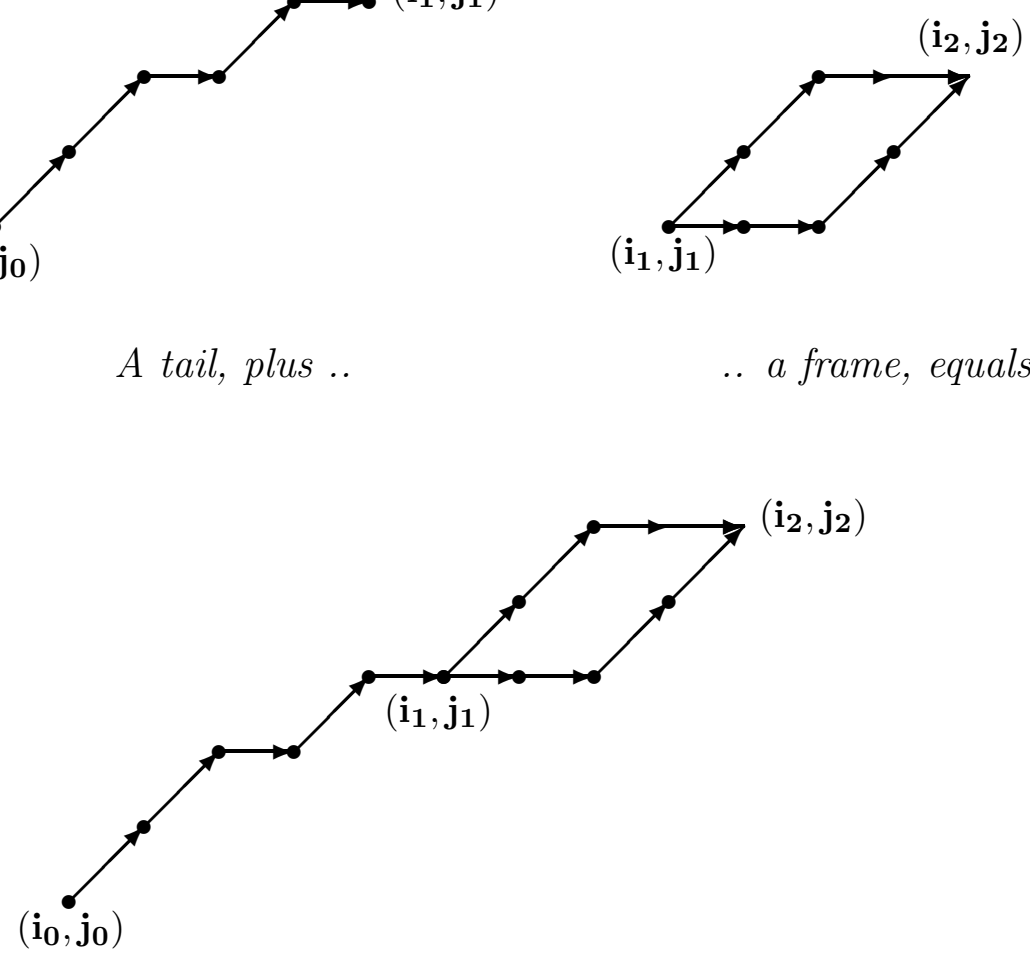

Figure 2: A kite 
two identical paths between these endpoints. The length of a tail is the number of vertices in the tail minus one, i.e., the number of edges.

A pair $\left(\omega_{1}, \omega_{2}\right)$ such that $\omega_{2}$ does not cross $\omega_{1}$ forms an alternating sequence of tails and frames, beginning with a tail. Note that tails may have length zero.

The upper tip of the final frame in this sequence is the common endpoint for two paths which intersect only at this common endpoint (these are the "ribbons" described above). One path ends at $(n, d)$, this is the top ribbon, and the other ends at $\left(n, d^{\prime}\right)$, the bottom ribbon.

Below we compute the probability of a frame from $\left(i_{1}, j_{1}\right)$ to $\left(i_{2}, j_{2}\right)$, a tail from $\left(i_{1}, j_{1}\right)$ to $\left(i_{2}, j_{2}\right)$, and a pair of ribbons with common initial point $\left(k, d^{\prime \prime}\right)$ and terminal points at $(n, d)$ and $\left(n, d^{\prime}\right)$, respectively.

Let $f_{\left(i_{1}, j_{1}\right)}^{\left(i_{2}, j_{2}\right)}(d)$ denote the probability of a frame from $\left(i_{1}, j_{1}\right)$ to $\left(i_{2}, j_{2}\right)$. Note for $f_{\left(i_{1}, j_{1}\right)}^{\left(i_{2}, j_{2}\right)}(d) \neq$ 0 , we must have $i_{2} \geq i_{1}+2, j_{2}>j_{1}$, and $j_{2}-j_{1} \leq i_{2}-i_{1}-1$. Assuming these conditions, we write

$$
f_{\left(i_{1}, j_{1}\right)}^{\left(i_{2}, j_{2}\right)}(d)=\sum_{(\alpha, \beta)} P(\alpha) P(\beta)
$$

where $(\alpha, \beta)$ is a pair of paths from $\left(i_{1}, j_{1}\right)$ to $\left(i_{2}, j_{2}\right)$ intersecting only at the endpoints such that $\beta$ does not cross $\alpha$ (i.e., $\alpha$ forms the upper edge of the frame, and $\beta$ forms the lower edge).

We convert the sum above into a determinant using the Gessel-Viennot thereom. Evaluation of the determinant gives

$f_{\left(i_{1}, j_{1}\right)}^{\left(i_{2}, j_{2}\right)}(d)=\frac{j_{1} j_{2}\left(d-j_{1}\right) !^{2}}{d^{2\left(i_{2}-i_{1}\right)}\left(j_{2}-j_{1}\right) !^{2}\left(d-j_{2}\right) !^{2}} \sum_{l, m=j_{1}}^{j_{2}}(-1)^{l+m}(l m)^{i_{2}-i_{1}-2}\left(l-j_{1}\right)\left(m-j_{2}\right)\left(\begin{array}{c}j_{2}-j_{1} \\ m-j_{1}\end{array}\right)\left(\begin{array}{c}j_{2}-j_{1} \\ l-j_{1}\end{array}\right)$

We compute the probability $t_{\left(i_{1}, j_{1}\right)}^{\left(i_{2}, j_{2}\right)}(d)$ of a tail from $\left(i_{1}, j_{1}\right)$ to $\left(i_{2}, j_{2}\right)$ in a manner analogous to the computation of $\sum_{\alpha} P(\alpha)^{2}$ in section 2.6. We obtain

$$
t_{\left(i_{1}, j_{1}\right)}^{\left(i_{2}, j_{2}\right)}(d)=\frac{\left(d-j_{1}\right) !^{2}}{d^{2\left(i_{2}-i_{1}\right)}\left(2 j_{2}\right) !\left(d-j_{2}\right) !^{2}} \sum_{m=1}^{j_{2}}(-1)^{j_{2}-j_{1}} m^{2\left(i_{2}-i_{1}\right)}(2 m) !\left(\begin{array}{c}
2 j_{2} \\
j_{2}+m
\end{array}\right)\left(\begin{array}{c}
m+j_{1}-1 \\
j_{1}-m
\end{array}\right)
$$

Finally we compute the probability $r\left(d, d^{\prime}, d^{\prime \prime}, k, n\right)$ of a pair of ribbons with common initial point $\left(k, d^{\prime \prime}\right)$ and terminal points $\left(n, d^{\prime}\right)$ and $(n, d)$. The probability is given by a determinant similar to the one in (10). In the present case, we have $d^{\prime \prime}$ in place of $d_{1}$ and $d^{\prime}$ in place of $d_{2}$. Thus

$$
r\left(d, d^{\prime}, d^{\prime \prime}, k, n\right)=\operatorname{det}\left(d, d^{\prime}, d^{\prime \prime}, k, n\right)
$$




\section{The "double dixie-cup problem," of Newman and Shepp, revisited}

Here we consider a different generalization of the coupon collector's problem. Let integers $h, d \geq 1$ be fixed. Again we are sampling with replacement from $d$ kinds of coupons, but now $T$ is the epoch at which we have collected at least $h$ copies of each of the $d$ coupons, for the first time (for example, my $h-1$ siblings and I might each want to have our own copy of every one of the available baseball cards). We study the expectation, the probability generating function, and the asymptotic behavior of the expectation, of this generalized problem.

These questions were investigated by Newman and Shepp [5] and the asymptotics were refined by Erdös-Rényi [2]. It is interesting to note that this problem is equivalent to one about the evolution of a random graph. Suppose we fix $n$ vertices, and then we begin to collect from among $n$ kinds of coupons. If we collect a particular sequence, say, $\left\{c_{1}, c_{2}, c_{3}, \ldots\right\}$ then we add the edges $\left(c_{1}, c_{2}\right),\left(c_{3}, c_{4}\right) \ldots$ That is, we add an edge each time we choose a new pair of coupons. Our problem about collecting at least $h$ copies of each kind of coupon is thereby equivalent to the question of obtaining a minimum degree of at least $h$ in an evolving random graph. ${ }^{1}$ In this section we will not add anything new to the asymptotics of this problem. Instead we claim only a simpler derivation than the original, and an explicit generating function, which gives a nice road to the asymptotics. We deal only with generating functions in one variable, whereas in [5] multivariate generating functions were used. We obtain not only the expectation of the time to reach a collection that has at least $h$ copies of each kind of coupon, but also the complete probability distribution of that time.

For $n$ fixed, consider a sequence of $n$ drawings of coupons that constitutes, for the first time at the $n$th drawing, a complete collection of at least $h$ copies of each of the $d$ kinds of coupons.

There are $d$ possibilities for the coupon that completes the collection on the $n$th drawing. There are $\left(\begin{array}{l}n-1 \\ h-1\end{array}\right)$ ways to choose the set of earlier drawings on which that last coupon type occurred. On the remaining $n-h$ drawings we can define, as usual, an equivalence relation: two drawings $i, j$ are equivalent if the same kind of coupon was drawn at the $i$ th and the $j$ th drawings. The number of such equivalence relations is equal to the number of ordered partitions of a set of $n-h$ elements into $d-1$ classes, each class containing at least $h$ elements. We will denote this latter number by $(d-1) !\left\{\begin{array}{c}n-h \\ d-1\end{array}\right\}_{h}$, where the $\left\{\begin{array}{l}n \\ k\end{array}\right\}_{h}$ 's count the unordered partitions of an $n$-set into $k$ classes of at least $h$ elements each.

The number of sequences of $n$ drawings for which we achieve a complete collection for

\footnotetext{
${ }^{1}$ Our thanks to Ed Bender and to a helpful referee for pointing this out.
} 
the first time at the $n$th drawing is therefore

$$
d\left(\begin{array}{l}
n-1 \\
h-1
\end{array}\right)(d-1) !\left\{\begin{array}{l}
n-h \\
d-1
\end{array}\right\}_{h} .
$$

Since there are $d^{n}$ possible drawing sequences of length $n$, the probability that $T=n$ is

$$
p_{n}=\frac{d !}{d^{n}}\left(\begin{array}{l}
n-1 \\
h-1
\end{array}\right)\left\{\begin{array}{l}
n-h \\
d-1
\end{array}\right\}_{h}
$$

and the probability generating function is

$$
\begin{aligned}
P_{h}(x) & \stackrel{\text { def }}{=} \sum_{n \geq 0} p_{n} x^{n}=\sum_{n \geq 0} \frac{d !}{d^{n}}\left(\begin{array}{l}
n-1 \\
h-1
\end{array}\right)\left\{\begin{array}{l}
n-h \\
d-1
\end{array}\right\}_{h} x^{n} \\
& =d !\left(\begin{array}{c}
x D-1 \\
h-1
\end{array}\right) \sum_{n \geq 0}\left\{\begin{array}{l}
n-h \\
d-1
\end{array}\right\}_{h}\left(\frac{x}{d}\right)^{n},
\end{aligned}
$$

where $D=\partial / \partial x$.

It remains to find the ordinary power series generating function of the $\left\{\begin{array}{l}n \\ k\end{array}\right\}_{h}$ 's. The exponential formula gives us immediately their exponential generating function, as

$$
\sum_{n \geq 0}\left\{\begin{array}{l}
n \\
k
\end{array}\right\} \frac{x^{n}}{n !}=\frac{1}{k !}\left(e^{x}-1-x-\ldots-\frac{x^{h-1}}{(h-1) !}\right)^{k}
$$

We can convert this into an ordinary power series generating function by applying the Laplace transform operator

$$
\int_{0}^{\infty} e^{-s x} \cdots d x
$$

to both sides, which yields

$$
\sum_{n \geq 0}\left\{\begin{array}{l}
n \\
k
\end{array}\right\}_{h} \frac{1}{s^{n+1}}=\frac{1}{k !} \int_{0}^{\infty} e^{-s x}\left(e^{x}-1-x-\ldots-\frac{x^{h-1}}{(h-1) !}\right)^{k} d x
$$

or finally

$$
\sum_{n \geq 0}\left\{\begin{array}{l}
n \\
k
\end{array}\right\}_{h} t^{n}=\frac{1}{k ! t} \int_{0}^{\infty} e^{-x / t}\left(e^{x}-1-x-\ldots-\frac{x^{h-1}}{(h-1) !}\right)^{k} d x .
$$

Now if we substitute (32) into (30) we obtain the probability generating function of the generalized coupon collector's problem in the form

$$
P_{h}(x)=\frac{1}{d^{h-2}} \int_{0}^{\infty}\left\{\left(\begin{array}{c}
x D-1 \\
h-1
\end{array}\right) x^{h-1} e^{-t d / x}\right\}\left(e^{t}-1-t-\ldots-\frac{t^{h-1}}{(h-1) !}\right)^{d-1} d t
$$


In the above, $\left(\begin{array}{c}x D-1 \\ h-1\end{array}\right)$ is the differential operator that is defined by

$$
\left(\begin{array}{c}
x D-1 \\
h-1
\end{array}\right) f(x)=\frac{1}{(h-1) !}\left(x \frac{d}{d x}-1\right)\left(x \frac{d}{d x}-2\right) \ldots\left(x \frac{d}{d x}-h\right) f(x) .
$$

However, it is easy to establish, by induction on $h$, the interesting fact that

$$
\left(\begin{array}{c}
x D-1 \\
h-1
\end{array}\right) x^{h-1} e^{-t d / x}=\frac{(t d)^{h-1}}{(h-1) !} e^{-t d / x} .
$$

Hence we have proved the following evaluation.

Theorem 2 The probability generating function for the coupon collecting problem in which at least $h$ copies of each coupon are needed is given by

$$
P_{h}(x)=\frac{d}{(h-1) !} \int_{0}^{\infty} t^{h-1} e^{-t d / x}\left(e^{t}-1-t-\ldots-\frac{t^{h-1}}{(h-1) !}\right)^{d-1} d t .
$$

\subsection{Two examples}

Let's look at the cases $h=1$, the classical case, and $h=2$, where we want to collect at least two specimens of each of the $d$ kinds of coupons.

If $h=1$ then (35) takes the form

$$
P_{1}(x)=d \int_{0}^{\infty} e^{-t d / x}\left(e^{t}-1\right)^{d-1} d t
$$

If we expand the power of $\left(e^{t}-1\right)$ by the binomial theorem and integrate termwise we obtain

$$
P_{1}(x)=x d \sum_{j=0}^{d-1}\left(\begin{array}{c}
d-1 \\
j
\end{array}\right) \frac{(-1)^{d-1-j}}{d-j x},
$$

which is precisely the partial fraction expansion of the classical generating function (3).

To see something new, let $h=2$. Then

$$
P_{2}(x)=d \int_{0}^{\infty} t e^{-t d / x}\left(e^{t}-1-t\right)^{d-1} d t .
$$

Again, by termwise integration this can be made fairly explicit, but since the most interest attaches to the expectation, let's look at the average number of trials that are needed to collect at least two samples of each of $d$ coupons. This is $P_{2}^{\prime}(1)$, which after some simplification takes the form

$$
P_{2}^{\prime}(1)=d^{2} \int_{0}^{\infty}\left(\frac{t^{2}}{e^{t}-1-t}\right)\left(1-(1+t) e^{-t}\right)^{d} d t
$$


From this we can go in either of two directions, an exact evaluation or an asymptotic approximation. By termwise integration it is easy to obtain the following exact formula, which is a finite sum, for $\langle T\rangle_{2}$, the average number of trials needed to collect at least two of each of the $d$ kinds of coupons:

$$
\langle T\rangle_{2}=d^{2} \sum_{m, j}(-1)^{m}\left(\begin{array}{c}
d-1 \\
m
\end{array}\right)\left(\begin{array}{c}
m \\
j
\end{array}\right) \frac{(j+2) !}{(m+1)^{j+3}}
$$

For $d=2,3,4,5$ these are $2,11 / 2,347 / 36,12259 / 864$. To facilitate comparison with the classical $(h=1)$ case, we show below, for $1 \leq d \leq 10$, a table of the expected numbers of trials needed when $h=1,2$.

$\begin{array}{rcccccccccc}d: & 1 & 2 & 3 & 4 & 5 & 6 & 7 & 8 & 9 & 10 \\ \langle T\rangle_{1}: & 1.0000 & 3.0000 & 5.5000 & 8.3333 & 11.417 & 14.700 & 18.150 & 21.743 & 25.460 & 29.290 \\ \langle T\rangle_{2}: & 2.0000 & 5.5000 & 9.6389 & 14.189 & 19.041 & 24.134 & 29.425 & 34.885 & 40.492 & 46.230\end{array}$

\subsection{Asymptotics}

Now we investigate the asymptotic behavior of (37), for large $d$, to compare it with the $d \log d$ behavior of the classical case where $h=1$.

Theorem 3 If there are d different kinds of coupons, and if at each step we sample one of the $d$ kinds with uniform probability, let $\langle T\rangle_{h}$ denote the average number of samples that we must take until, for the first time, we have collected at least $h$ specimens of each of the $d$ kinds of coupons. Then for every $h \geq 1$, we have $\langle T\rangle_{h} \sim d \log d \quad(d \rightarrow \infty)$.

Consider first the case $h=2$. In (37) we make the substitution

$$
e^{-u}=1-(1+t) e^{-t},
$$

where $u$ is a new variable of integration. We then find that

$$
P_{2}^{\prime}(1)=d^{2} \int_{0}^{\infty} t(u) e^{-u d} d u
$$

where $t(u)$ is the inverse function of the substitution (39), which is well defined since the right side of (39) increases steadily from 0 to 1 as $t$ increases from 0 to $\infty$.

The main contribution to $P_{2}^{\prime}(1)$ comes from values of $u$ near $u=0$, and when $u$ is near 0 we have

$$
t(u)=-\log u+O(\log \log u) .
$$


Following the arguments in [7, sec. 2.2, we see that $P_{2}^{\prime}(1)$ of (40) has the same asymptotic behavior as

$$
d^{2} \int_{0}^{c}(-\log u) e^{-u d} d u, \quad(0<c<1)
$$

and in [7] this is shown to be

$$
\sim d^{2} \cdot \frac{\log d}{d}=d \log d
$$

Now we consider the asymptotic behavior of the expected number of trials for general values of $h$. From (35) we see that this expected number of trials can be written in the form

$$
\frac{d^{2}}{(h-1) !} \int_{0}^{\infty}\left\{\frac{t^{h}}{e^{t}-1-t-\ldots-\frac{t^{h-1}}{(h-1) !}}\right\}\left\{1-\left(1+t+\frac{t^{2}}{2}+\ldots+\frac{t^{h-1}}{(h-1) !}\right) e^{-t}\right\}^{d} d t
$$

Again we make the change of variable

$$
e^{-u}=1-\left(1+t+\frac{t^{2}}{2}+\ldots+\frac{t^{h-1}}{(h-1) !}\right) e^{-t}
$$

in the integral, and it takes the remarkably simple form (compare (40))

$$
P_{h}^{\prime}(1)=d^{2} \int_{0}^{\infty} t(u) e^{-u d} d u
$$

where $t(u)$ is the inverse function of the substitution (42). Again the main contribution to the integral comes from small values of $u$, and when $u$ is small and positive we have

$$
t(u)=-\log u+(h-1) \log (-\log u)+\ldots
$$

Using the method of sec. II.2 of [7] once more, we find that

$$
\langle T\rangle_{h}=d \log d+(h-1) d \log \log d(1+o(1)) \quad(d \rightarrow \infty) .
$$

We remark that in the case of $d=200$ coupons, the correct expected number of trials to obtain two of each coupon is 1614 trials, the approximation $d \log d$ is 1175 , and the approximation $d \log d+(h-1) d \log \log d$ is 1393, each rounded to the nearest integer.

\section{The number of singletons}

In view of the asymptotics in the preceding section we realize that at the moment when a coupon collector sequence terminates with a complete collection, 'most' coupons will have 
been collected more than once, and only 'a few' will have been collected just once. We call a coupon that has been seen just once a singleton. We will now look at the distribution of singletons.

In more detail, let $j$ be the number of singletons in a collecting sequence that terminates successfully at the $n$th step. We first want the joint distribution $f(n, j)$ of $n$ and $j$, i.e., the probability that a collecting sequence halts successfully at the $n$th step, and has exactly $j$ singletons at that moment. We claim that

$$
f(n, j)=\frac{d !}{d^{n}}\left(\begin{array}{l}
n-1 \\
j-1
\end{array}\right)\left\{\begin{array}{l}
n-j \\
d-j
\end{array}\right\}_{2}
$$

Indeed, the last coupon to be collected can be chosen in $d$ ways, the other $j-1$ singleton coupons can be chosen in $\left(\begin{array}{l}d-1 \\ j-1\end{array}\right)$ ways, and can be presented in an ordered sequence in $(j-1)$ ! $\left(\begin{array}{c}d-1 \\ j-1\end{array}\right)$ ways. This ordered sequence can appear among the first $n-1$ trials in $\left(\begin{array}{c}n-1 \\ j-1\end{array}\right)$ ways, and the remaining $n-j$ trials constitute an ordered partition of $n-j$ elements into $d-j$ classes, no class having fewer than two elements, which can be chosen in $(d-j) !\left\{\begin{array}{l}n-j \\ d-j\end{array}\right\}_{2}$ ways. If we multiply these together and divide by $d^{n}$, the number of $n$-sequences, we obtain the result (44) claimed above.

Next we compute the probability that a completed collecting sequence contains exactly $j$ singletons, whatever the length of the sequence may be. That is we find $F(j)=\sum_{n} f(n, j)$, where $f$ is given by (44). We have, after using the generating function (32),

$$
\begin{aligned}
F(j) & =\sum_{n} \frac{d !}{d^{n}}\left(\begin{array}{c}
n-1 \\
j-1
\end{array}\right)\left\{\begin{array}{l}
n-j \\
d-j
\end{array}\right\}_{2} \\
& =\frac{d !}{(d-j) ! d^{j}}\left\{\int_{0}^{\infty}\left\{\left(\begin{array}{c}
t \frac{\partial}{\partial t}+j-1 \\
j-1
\end{array}\right)\left(\frac{e^{-x t}}{t}\right)\right\}\left(e^{x}-1-x\right)^{d-j} d x\right\}_{t \rightarrow 1 / d}
\end{aligned}
$$

But using the fact that, analogously to (34), we have

$$
\left(\begin{array}{c}
t \frac{\partial}{\partial t}+j-1 \\
j-1
\end{array}\right)\left(\frac{e^{-x t}}{t}\right)=\frac{x^{j-1}}{(j-1) ! t^{j}} e^{-x / t},
$$

we can simplify the expression for $F(j)$ to

$$
F(j)=j\left(\begin{array}{l}
d \\
j
\end{array}\right) \int_{0}^{\infty} x^{j-1}\left(e^{x}-1-x\right)^{d-j} e^{-x d} d x, \quad(j=1,2,3, \ldots)
$$

which is the desired distribution of the number of singletons in a successfully terminated coupon collecting sequence. 
Now if we multiply by $j$ and sum over $j$, we'll get the average number of singletons that appear in a completed collection of $d$ coupons. This is, after some termwise integration,

$$
\bar{j}(d)=d \sum_{m}(-1)^{m}\left(\begin{array}{c}
d-2 \\
m
\end{array}\right) \frac{d(m+1)+1}{(m+2)^{2}(m+1)} .
$$

If we expand the summand in partial fractions, viz.,

$$
\bar{j}(d)=d \sum_{m}(-1)^{m}\left(\begin{array}{c}
d-2 \\
m
\end{array}\right)\left(\frac{1}{m+1}-\frac{1}{m+2}+\frac{d-1}{(m+2)^{2}}\right),
$$

then each of the three sums indicated can be expressed in closed form, in two cases by using the identity

$$
\sum_{k}(-1)^{k}\left(\begin{array}{l}
n \\
k
\end{array}\right) \frac{1}{x+k}=\frac{1}{x\left(\begin{array}{c}
x+n \\
n
\end{array}\right)},
$$

directly, with $x=1$ and $x=2$, and in the third case by differentiating (48) w.r.t. $x$, and using the result with $x=2$. The identity (48) is itself certified, after multiplying by the denominator on the right, by the WZ proof certificate $R(n, k)=k(x+k) /((n+1)(k-n-1))$.

What results is that $\bar{j}(d)=H_{d}$, the $d$ th harmonic number. That is, the average number of singleton coupons in a completed collection sequence of d coupons is the harmonic number $H_{d}$.

\section{References}

[1] Louis Comtet, Advanced Combinatorics, Reidel, 1974.

[2] P. Erdős, and A. Rényi, On a classical problem of probability theory. (English. Russian summary) Magyar Tud. Akad. Mat. Kutat Int. Kzl. 6 (1961), 215-220.

[3] L. Flatto, Limit theorems for some random variables associated with urn models. Ann. Probab. 10 (1982), no. 4, 927-934.

[4] Ira Gessel and Gérard Viennot, Binomial determinants, paths, and hook length formulae, Adv. in Math. 58 (1985), no. 3, 300-321.

[5] Donald J. Newman and Lawrence Shepp, The double dixie cup problem, Amer. Math. Monthly 67 (1960), 58-61.

[6] Herbert S. Wilf, generatingfunctionology, Academic Press, second ed. 1994.

[7] R. Wong, Asymptotic approximations of integrals, Academic Press, Boston, 1989. 